\title{
Improved field scanner incorporating parabolic optics. Part 1: Simulation
}

\author{
Galiya Sharafutdinova, ${ }^{1}$ John Holdsworth, ${ }^{1, *}$ and Dirk van Helden ${ }^{2}$ \\ ${ }^{1}$ School of Mathematical and Physical Sciences, University of Newcastle, \\ University Drive, Callaghan, NSW, 2308, Australia \\ ${ }^{2}$ School of Biomedical Sciences, University of Newcastle, \\ University Drive, Callaghan, NSW, 2308, Australia \\ ${ }^{*}$ Corresponding author: john.holdsworth@ newcastle.edu.au
}

Received 11 March 2009; revised 21 May 2009; accepted 10 July 2009; posted 10 July 2009 (Doc. ID 108634); published 23 July 2009

\begin{abstract}
An unobstructed afocal scanning system design employing two off-axis parabolic reflectors as relay optics between two flat scan mirrors is proposed and investigated using OSLO optical software. It is found that, with a symmetric arrangement of the parabolic reflectors and appropriate selection of the first scan mirror rotational axis, the system provides linear scan lines at the image surface and excellent point spread function results in all scan positions. The design is functionally equivalent to a single-mirror scan engine and superior in every metric to a comparable dimension spherical mirror arrangement. This design is suited to two-dimensional laser scan engines and for confocal and two-photon microscopy in particular. (c) 2009 Optical Society of America
\end{abstract}

OCIS codes: $\quad 080.4035,080.4228,080.6755,170.5810,180.5810$.

\section{Introduction}

The noble gas lasers exhibit extremely good collimation of their output due to their resonator configuration [1], and this feature of lasers has been employed in laser scanning widely since the late 1960s. Linearity of scan is important for many applications and the design of scan engines continues to be an active area of research [2-4] . Polygon-mirror-based scanners have undergone much development [5] and are employed in industrial line scanning app $\bar{i}-$ cations over fixed areas. Galvanometer-mounted mirrors allow adjustment in size of the laser scanned region and this beneficial feature has led to laser scanning in this manner being widely deployed in microscopy, especially confocal microscopy as proposed by Minsky [6] and the subsequent variants of the technique. The whole system specification may be determined by the performance of the two-dimensional laser scanning system used prior to the microscope

0003-6935/09/224389-08\$15.00/0

(C) 2009 Optical Society of America proper, and there are numerous approaches to laser scanning of the beam across the sample $[2,4,7-10]$.

The simplest approach is to use a single mirror, gimballing about a point on its surface, to scan the beam across a target area. However, this remains impractical as the fast and precise control of an appropriate mount is not commercially available. The next most simple scanning system of two close-coupled scan mirrors is limited in speed, defined by the size, or strictly, the moment of inertia of the second scan mirror, though this can be improved by using a paddle first scan mirror $[8,9]$ or building a golf-club scanner [8]. The pincushion effect, and lateral elongation created in the scanning pattern of the simple twomirror scanners, may be compensated by scan lenses [11] or imaging software.

Relay optics may be used between the two deflecting mirrors to keep the size and mass of both scan mirrors small so that imaging speed can be increased. Lens relay optics introduce limits to optical performance due to chromatic aberrations as well as temporal and spatial distortions in ultrashort pulses used in multiphoton microscopy. The best approach 
is to use reflecting optics as achromatic relay optics between the two scanning mirrors. A scan engine including four reflecting elements, comprising two flat scanning mirrors and two spherical reflectors, was designed by Amos [7] and employed in the BioRAD confocal microscopy scanning systems as the "gold standard" in scanning microscopy, while Denk patented a similar optical system as a scan engine in a two-photon microscopy system [12] well prior to the ultrashort-pulse-based systems currently available. The two spherical mirrors within these systems are in a confocal arrangement and are necessarily tilted to avoid obstructing the beam, as detailed in Fig. 1 and, hence, the degree of spherical aberration affecting a beam propagating through the scanner is increased. Spherical aberrations in the focusing reflecting elements can be avoided by using the off-axis section of a parabolic surface instead of spherical mirror surfaces. We propose a design of an afocal scan engine based on two parabolic optical elements placed in the mirror train as relay optics.

The properties of a parabola were defined by the ancient Greeks. The optical performance advantages have been periodically rediscovered [13] due to the fact that a parabolic surface can reflect light from infinity to an unobstructed point focus with minimal aberration. Specialized applications of parabolic reflectors include both focusing and afocal telescopes [14-21], most with large to very large apertures, and capture of large solid angle femtosecond fluorescence without optical aberrations modifying the temporal behavior of the fluorescence [22]. To the best of our knowledge, parabolic optics have not been considered as elements in a four-mirror scanning system. Most likely this is principally due to cost, but is also because of the aberrations and coma that arise when parabolas focus light rays from a finite distance, factors that have ensured that spherical reflectors dominate as the reflectors of choice in commercial confocal and two-photon laser scanning microscope systems [7,12,23].

The scope of this paper is to model and examine the performance of afocal scan engines prior to any microscope. The coupling of these engines into the microscope objective conjugate plane reveals the extent of focused beam distortion. However, as this involves other microscope-specific optical elements, this paper is limited to discussion of the scan engine design itself. OSLO optical software (Lambda Research Corporation) has been used to design and simulate the performance of the two comparable four-mirror scan engines and a reference system of a single gimballing mirror. The scan engines are compared to each other in terms of the point spread function, the spot size of a Gaussian beam, and the flatness of the scan lines in the image plane.

\section{Model Design}

The simulated model designs of the three scan engines in this paper are of a single flat reflector reference system (RS) and two different fourreflector afocal optical relays comprising two flat scanning mirrors and two concave surfaces, which are

- the Amos [7] scan engine as detailed in Fig. 1. It features two static spherical surfaces as relay optics between the two galvanometer-driven flat scanning mirrors. This design is labeled as the spherical scan engine (SSE) in further discussion and is used to compare properties with the new system design.

- the new parabolic scan engine design, which incorporates two static off-axis parabolic mirrors as relay optics between the two flat galvanometer-driven scanning mirrors, as detailed in Fig. 2. This is labeled as the parabolic scan engine (PSE) in further discussion.

For all scan engines, the object, i.e., the laser beam waist, is positioned $2 \mathrm{~m}$ away from the first scanning mirror, the aperture stop is on the first scanning mirror surface, the image surface is located at infinity, as they are afocal systems, and the image aperture radius is $10 \mathrm{~mm}$ at a distance of $15 \mathrm{~mm}$ from the last scanning mirror. The aperture radius and distance were chosen to mimic the entrance aperture of a scan lens, although the effect of a refractive focusing element is not included in the paper. In all engine designs the scanning mirror surfaces move $\pm 10^{\circ}$ in each single axis, or both axes for RS, producing a $\pm 20^{\circ}$ line scan about the original zero position. With the four-mirror systems, the first mirror scans in the horizontal direction and the second mirror scans the vertical axis. The laser is modeled from idealized typical laser beam parameters of the Spectra-Physics Tsunami and Coherent Chameleon. The wavelength of the circular Gaussian intensity profile laser beam is $790 \mathrm{~nm}$ with $1 / e^{2}$ waist radius of $0.4 \mathrm{~mm}$ and halfangle divergence of $0.629 \mathrm{mRad}$ and an $M$-squared factor of 1 .

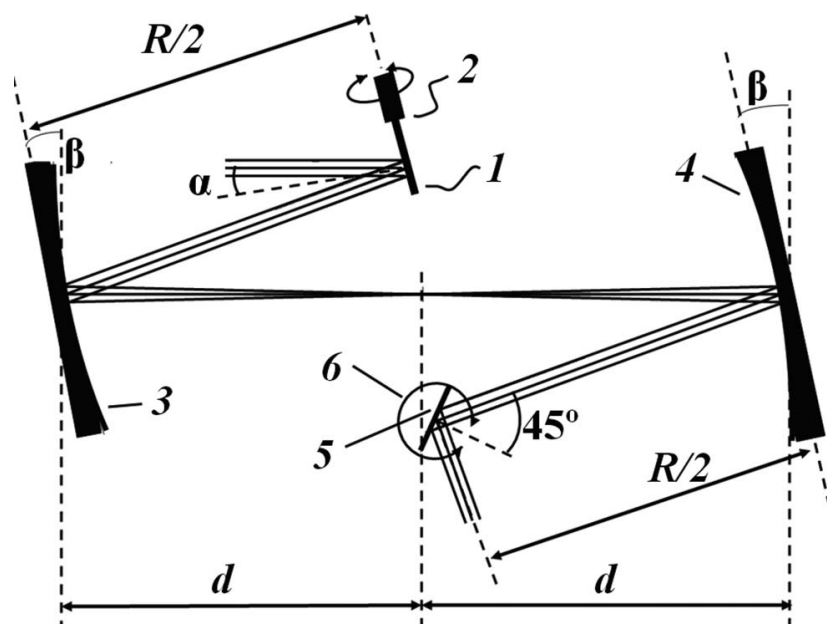

Fig. 1. Spherical-mirror-based achromatic scanning system after Amos [7]. 


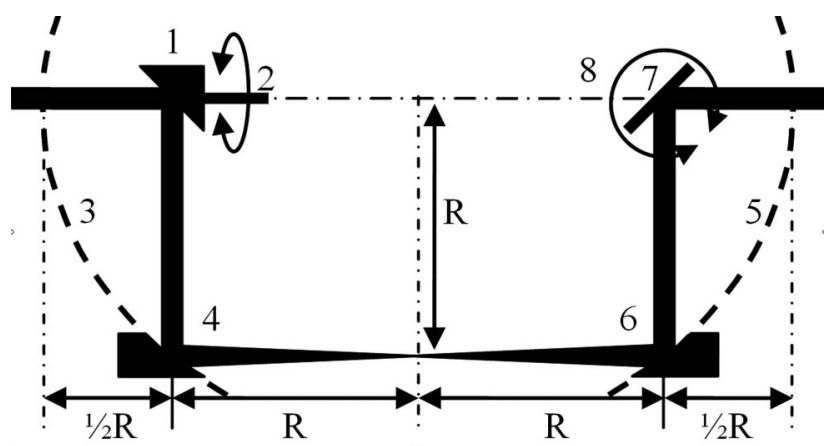

Fig. 2. Optical layout of the improved scanner design.

\section{A. Spherical Scan Engine System}

Parameters for simulating the SSE system are largely drawn from patent data [7] and are illustrated in Fig. 1. The two plane mirrors, 1 and 5, are driven by galvanometers deflecting the beam in two orthogonal directions. Mirror 1 rotates around axis 2, reflecting the incident beam to a scan line on spherical surface 3 . Mirror 4 gathers the light reflected from mirror 3 to one point on surface 5. Mirror 5 is rotated in the direction illustrated by line 6 about an axis that lies on its surface and is perpendicular to the page plane to create the two-dimensional scanning pattern.

The patent quotes a $75 \mathrm{~mm}$ spherical reflector focal length; however, to facilitate a better comparison with the $101.6 \mathrm{~mm}$ focal length parabolic reflectors, the spherical reflector focal length dimension chosen for simulation is also $101.6 \mathrm{~mm}$. This has the effect of slightly reducing the spherical aberrations of the spherical reflector system.

To minimize the scan line field curvature, Amos [24] found that plane mirrors 1 and 5 have to be oriented at an angle to each other. In this simulation, mirror 1 is tilted at the angle $\alpha=10^{\circ}$, as distinct from its rotation of $\pm 10^{\circ}$. Spherical surfaces 3 and 4 are tilted at an angle of $\beta=10^{\circ}$ relative to their respective incident beams to separate the incident and reflected beams and, thus, ensure that the focal point of reflector 3 is not vignetted. In general, the value of this angle depends on the geometric size of the reflectors chosen and the physical separation between the scanner mirrors. A smaller angle will introduce less spherical aberration in the system and less curvature of the scan lines, as discussed in Subsection 3.A.

The light reflected from mirror 3 is focused at an intermediate point, the position of which is found in OSLO by solving for the distance $d$ from mirror surface 3 to focus the diverging beam to the smallest spot. Spherical reflector 4 is positioned at distance $d$ from the intermediate point to minimize the aberration in the symmetrical optical system.

\section{B. Parabolic Scan Engine System}

The new scanning design is illustrated in Fig. 2. Parabolic reflectors 4 and 6 are the off-axis circular segment from parabolic surfaces 3 and 5, respectively, with radius of curvature $R$ and focal length
$R / 2$. Parabolic reflectors 4 and 6 are identical Newport Corporation off-axis replicated parabolic mirrors $50338 \mathrm{Al}$ and have an effective focal length of $101.6 \mathrm{~mm}$, which is also the radius of curvature of the surface, and a true focal length of $50.8 \mathrm{~mm}$. Use of identical parabolic reflectors is not strictly necessary, but allows a symmetrical optical system to be constructed and, therefore, aberrations introduced by the first concave surface, 4 , to be minimized and a uniform beam diameter to be maintained. The deflection axes of scanning mirrors 1 and 7 are positioned at the focal points of parabolic reflectors 4 and 6 , respectively. Mirrors 1 and 7 rotate around axis 2 and an axis lying on the surface of mirror 7, perpendicular to both rotation axis 2 and to the plane of this page. Line 8 demonstrates the direction of the mirror 7 movement. Mirror 1 is angled at $45^{\circ}$ to the incident beam and rotational axis 2 is coaxial with the incident beam.

This arrangement, and particularly the alignment and rotation of scan mirror 1 about axis 2, ensures that the beam traces a geometric circle on the surface of parabolic reflectors 4 and 6 and results in the beam impinging on the center of mirror 7 , independent of the scan deflection angle, within the limit of the aperture stop defined by the physical size $(38 \mathrm{~mm})$ of the surface 4 .

The distance from parabolic reflector 4 to reflector 6 is determined by the parabola's parameters and was held at $2 R$, as shown in Fig. 2 , due to the choice of a symmetric design. Parabolic reflector 4 focuses the beam at a midpoint due to the incident laser beam diameter. The exact position of the midpoint focus is determined by the beam divergence and will vary slightly from the distance $R$ to give a slight magnification of the beam size at mirror 7. Mirror 7 was modeled as a conventional galvanometer scanner rotating about an axis in the plane of the mirror surface.

\section{Results and Discussion}

Twenty-five scan field points as defined and shown in Fig. 3 have been used as the basis for calculations. These 25 points include zero and maximum deflection in each, and both axes and are representative of the complete frame in a scan pattern. In further discussion, these 25 points define separate optical configurations within OSLO and will be referred to as configurations.

\section{A. Shape of the Scan Lines}

The beam outlines in the image surface plane for the RS, SSE, and PSE scan systems are shown in Fig. 4 for each of the configurations. The spherical reflector SSE system exhibits curved scan lines at the final image surface, whereas the scan lines created by the RS have a slight pincushion distortion and the horizontal scan lines within the PSE system are straight and free of field distortion for any scan deflection angle, although there is some pincushion distortion in the vertical scan lines. 


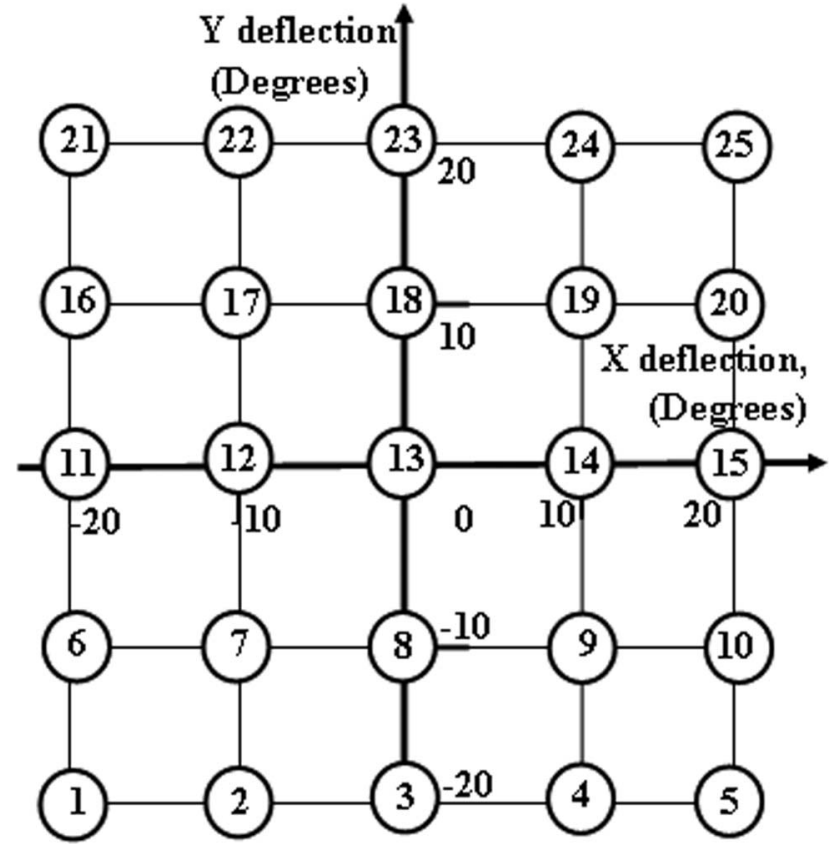

Fig. 3. Symmetrical raster scanning pattern illustrated by the black lines with 25 defined analysis points.

The PSE system gives a straight scan line because the deflection by the first scanning mirror, mirror 1 in Fig. 2, traces a circular arc on the surface of the first off-axis parabolic mirror with the radius equal to the curvature radius of the parabolic reflector at that line. Points on this line are subsequently reflected to the second parabolic reflector and form a similar radius circle segment. Finally, all these points are focused by the second parabolic reflector to its focal point, where the deflection point of the second scanning mirror is situated. Hence, the scan lines are straight.

The scan line curvature in the system SSE was found by Amos to rely on the orientation of the first scanning mirror to the incident beam. Analysis of the scan line field curvature for the SSE system in this paper yields that, for a fixed radius of curvature of the spherical reflectors used, the field curvature in the image plane depends most strongly on the tilt angle of the second spherical reflector, surface 4 in Fig. 1. In reality, this angle is defined by the distance between the two scanning mirror mounts and will be a factor defined by engineering choices.

\section{B. Beam Spot Size}

The scanning laser beam propagation parameters and the beam spot sizes are calculated by the astigmatic Gaussian beam analysis option within OSLO for the 25 configurations. The calculated results are displayed graphically in Fig. 5, where the groups of five configurations represent the horizontal scan lines. Configurations 11 through 15 represent the central scan line and exhibit the best performance, particularly in the center, as expected. Those data show that, in the PSE with parabolic reflectors and the reference system, the nondeflected beam spot in configuration 13 is circular and nonastigmatic. In the SSE system, the beam spot for configuration 13 is elongated in the horizontal direction and astigmatic. The beam tracking along a scan line becomes azimuthally turned, as evident in the footprints in Fig. $\underline{4}$, and this can be seen in the difference between the $x$ and $y$ spot radii for all systems and the increase or decrease in the beam size from the center position in a scan line toward the edges.

The deviation from a circular scanning beam spot is larger and increases to a greater extent along the scan lines for the SSE than for the PSE system. Moreover, in the SSE system there is a lack of symmetry between the $X$ extremity configurations, 11 and 15, and the $Y$ extremities, 3 and 23. All of these points are a $10^{\circ}$ mirror deflection from the central position, but the spherical system has different circularity due to the coma arising from the tilt of the spherical mirrors. This coma gives rise to the excellent circularity of the SSE system in configuration 18 , where there is a correction of the coma by the $5^{\circ}$ scan angle of the $y$-scan mirror. The magnitude of the deviation from circularity is approaching a factor of 2 worse for the spherical system than the
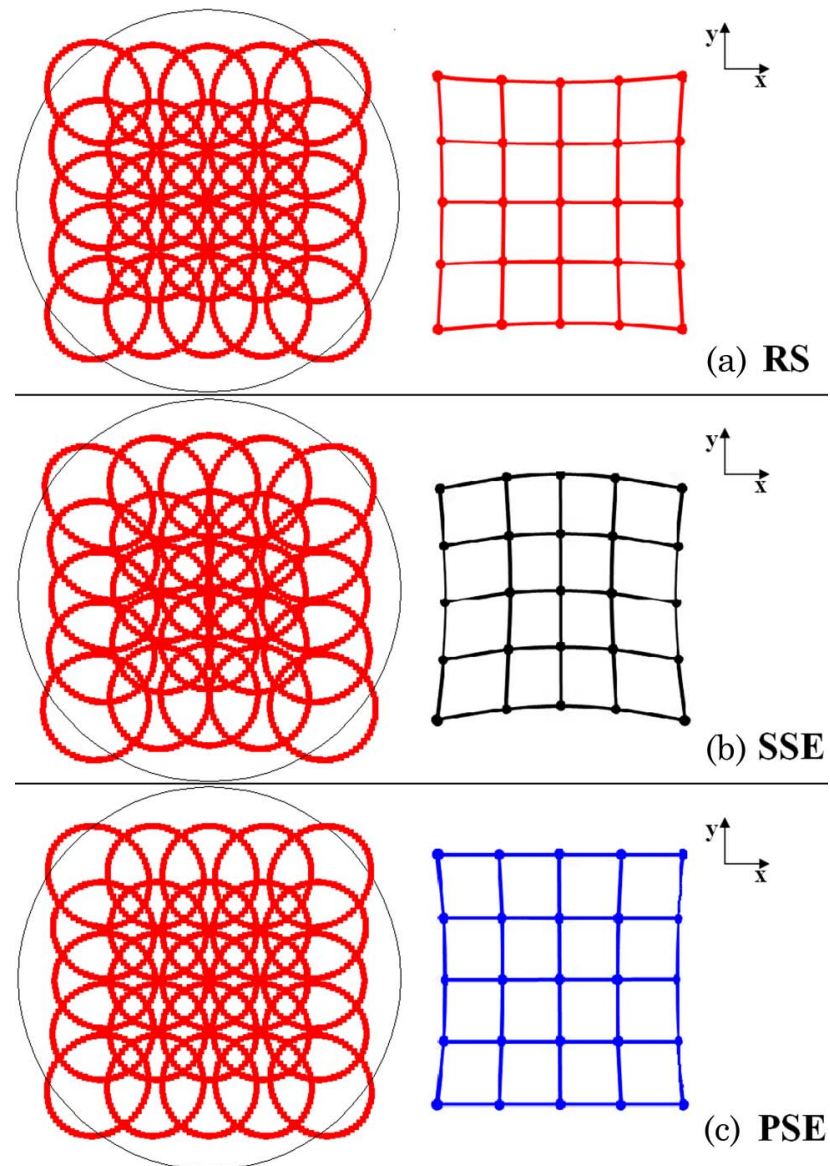

(c) PSE

Fig. 4. (Color online) Image surface scanned beam footprint and the scan field distortion for (a) the single-mirror reference system, (b) the spherical mirror system SSE, and. (c) the parabolic mirror system PSE. 


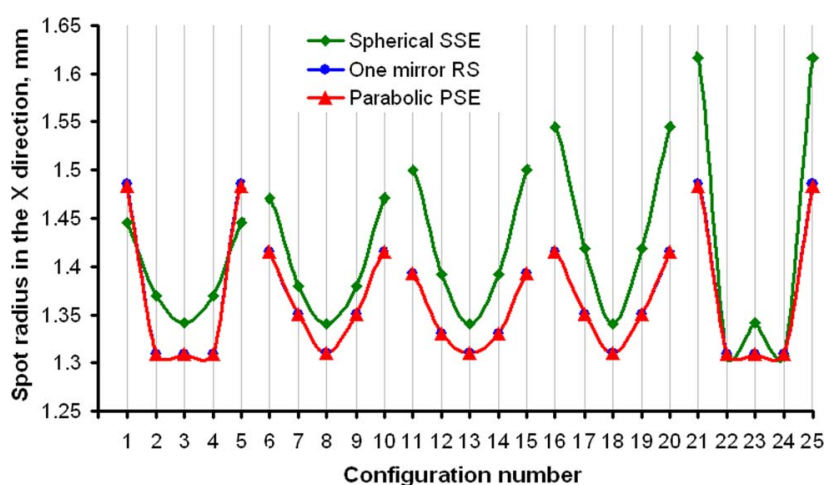

(a)

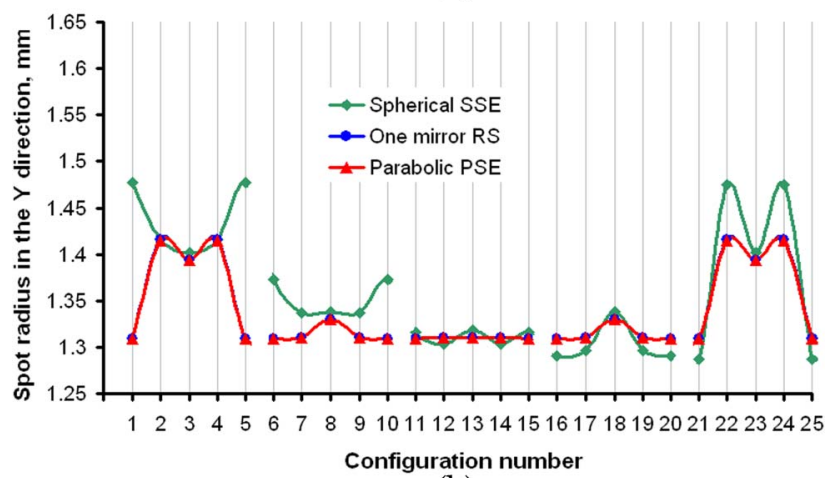

(b)

Fig. 5. (Color online) Comparison of the astigmatic beam spot size in the (a) $X$ and (b) $Y$ directions for the three scan systems, SSE, PSE, and RS. Configurations are subdivided into five groups, which refer to scan lines in the scan pattern. There is almost complete overlap of the PSE and RS results.

parabolic system, while the reference system and the parabolic system are almost identical.

\section{Spot Diagrams and Point Spread Functions}

The spot diagrams at infinity, as well as an outline of the Airy disk, for all 25 configurations in the RS, PSE, and SSE systems are shown in Figs. $\underline{6}-\underline{8}$, respectively, and highlight the differences between the scan engines rather well. The point spread functions (PSFs), also at infinity, for all systems are illustrated in Figs. 9-11. OSLO was configured to compute the spot diagram as an image of a single on-axis object point and the PSF as the diffraction image of the same on-axis object point in the generation of these images.

From the spot diagrams in Figs. $6-8$, it is evident that, for the SSE system, the image of the beam for zero scanning deflection, configuration 13 , is distorted due to coma in the vertical axis, and this extends to configurations $3,8,16$, and 19 , as well. As the scan moves horizontally from the center in the five scan lines, the spot size increases in the vertical direction and gradually increases in the horizontal direction. These results in Fig. 8 are in stark contrast to the PSE system with parabolic reflectors in Fig. 7 and the reference system of Fig. $\underline{6}$, where the spot size, as well as the shape, does not change substantially with deviation from the central position.

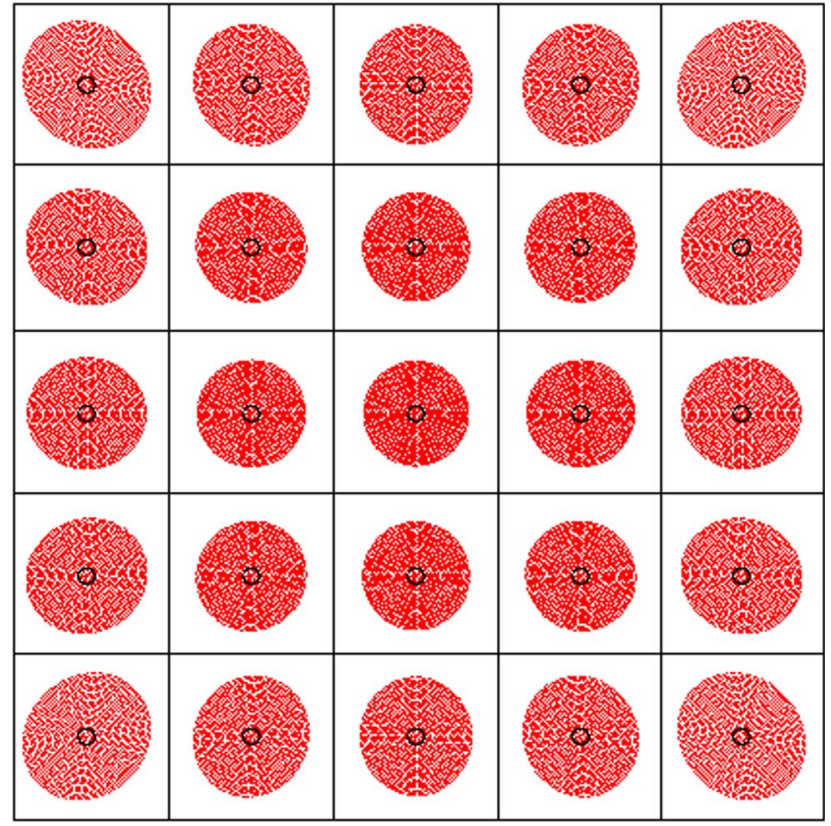

E ach individual square size is $4 \times 4 \mathrm{mRad}$

Fig. 6. (Color online) Spot diagram array for the $25 \mathrm{RS}$ system configurations examined. The black circle represents the Airy disk.

The point spread functions of each configuration and system in Figs. 9-11 highlight the stark difference in performance between the spherical and parabolic surfaces. These results are closely related to the spot diagram image of each configuration, of course, but do graphically emphasize the difference, or lack of, between the ideal reference system and the two

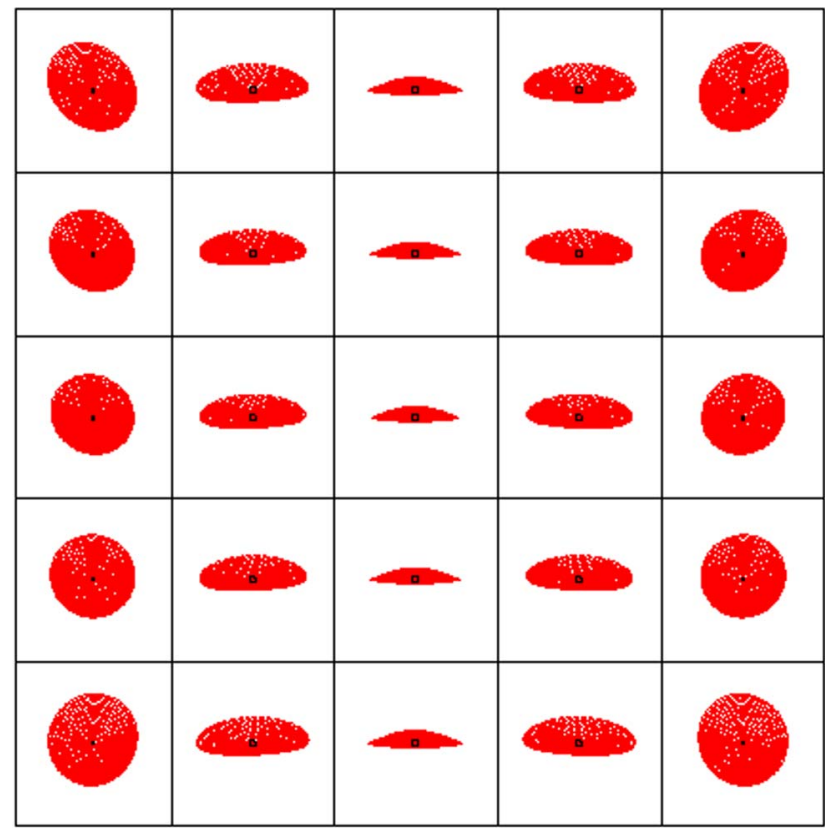

The size of the individual squares in the first and fifth columns is $20 \times 20 \mathrm{mRad}$, and for the rest is $10 \times 10 \mathrm{mRad}$

Fig. 7. (Color online) Spot diagram array for the 25 PSE system configurations examined. 


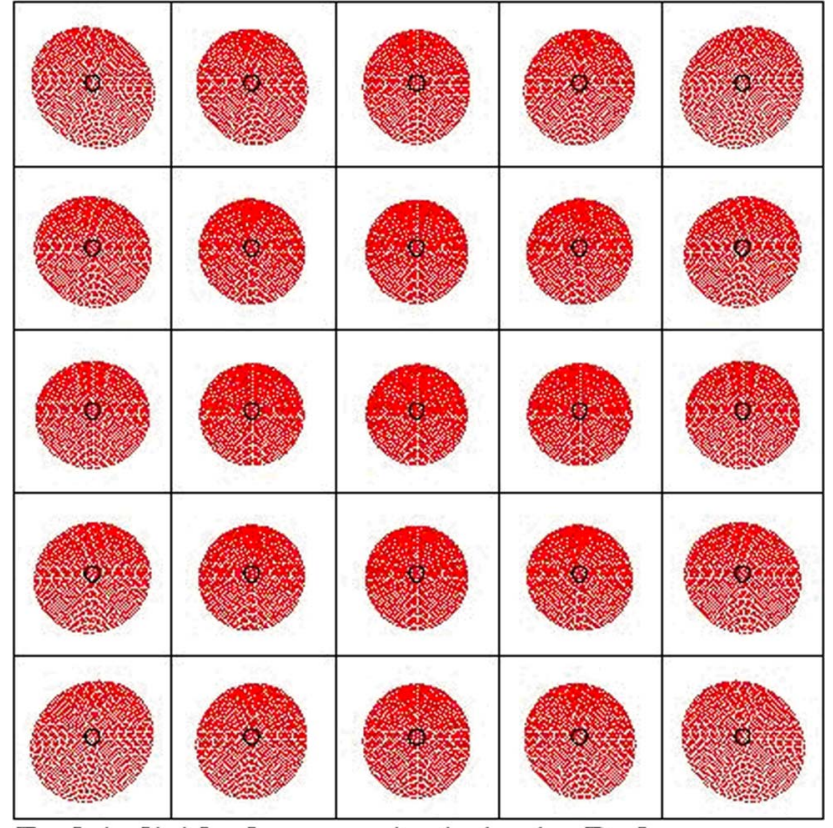

E ach individual square size is $4 \times 4 \mathrm{mRad}$

Fig. 8. (Color online) Spot diagram array for the 25 SSE system configurations examined. Note that the scale varies in comparison to Figs. $\underline{6}$ and $\underline{7}$ and also in columns 1 and 5 .

curved optic scan engines. The central amplitude of the point spread functions are analyzed graphically in Fig. 12. The amplitude at the peak of the point spread function has been measured against a normalized scale for all configurations, covering both four-mirror systems, and is remarkably constant in the parabolic mirror PSE system, only varying effectively in the fifth decimal place with the range of values falling within \pm 0.00009 . The spherical mirror equivalent, in contrast, varies from 0.013 to 0.073 , a range of \pm 0.03 and a factor of 5 .

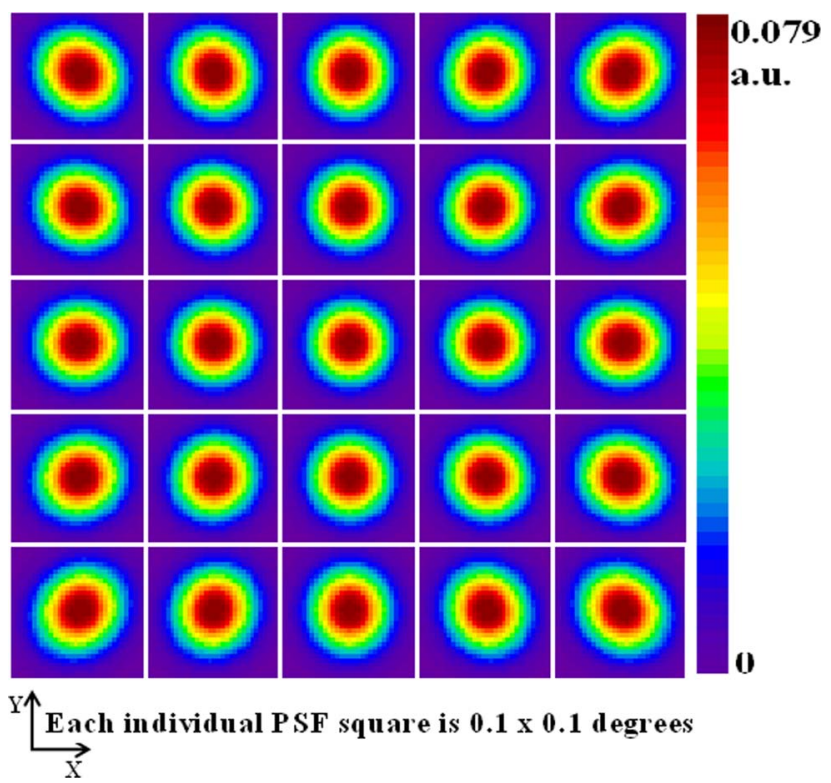

Fig. 9. (Color online) Point spread function data for the $25 \mathrm{RS}$ system configurations examined.

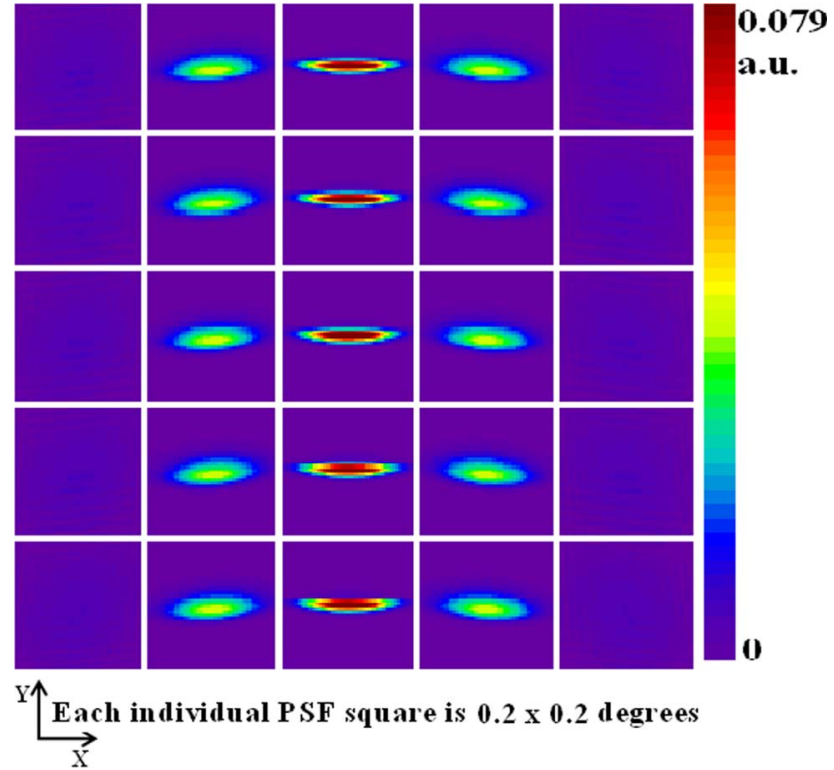

Fig. 10. (Color online) Point spread function data for the $25 \mathrm{SSE}$ system configurations examined.

\section{Comparison of Modeling Results with Specific Literature Results}

Howard and Stone [25] mathematically proved that, for the plane-symmetrical optical system with two spherical reflectors, the image of the point object without blur can be achieved when the object point, the centers of curvature of both mirrors, and the image point are all aligned on the axis of symmetry for the system. The models presented here confirm their conclusions, as this condition is not true for the SSE system and is true for the PSE system.

Reduced imaging aberrations in systems like SSE can be achieved by inserting additional tilted lenses in the beam path between the spherical reflectors

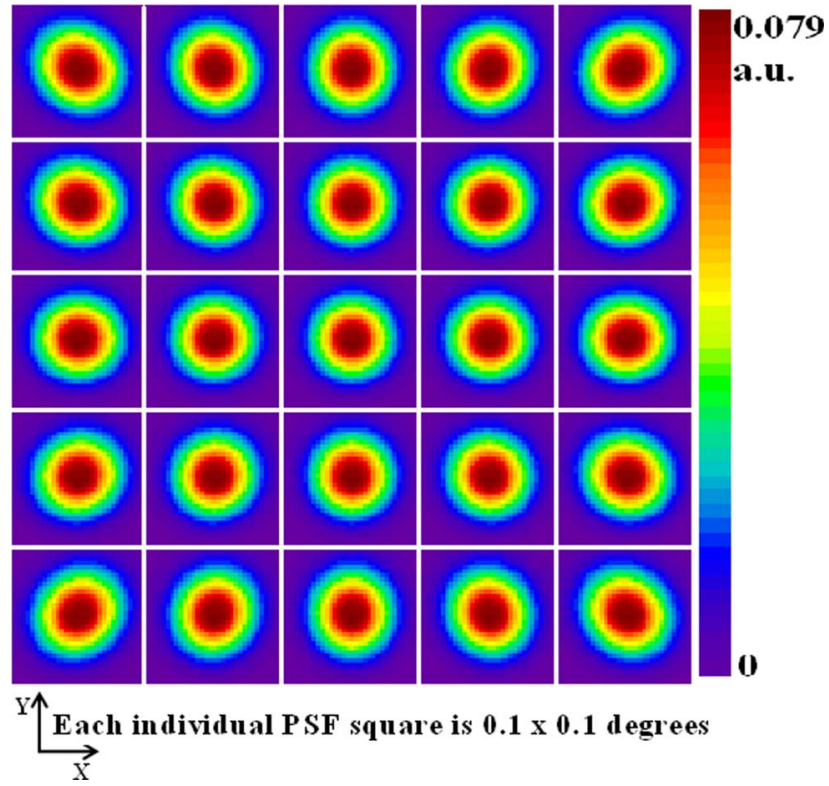

Fig. 11. (Color online) Point spread function data for the 25 PSE system configurations examined. 


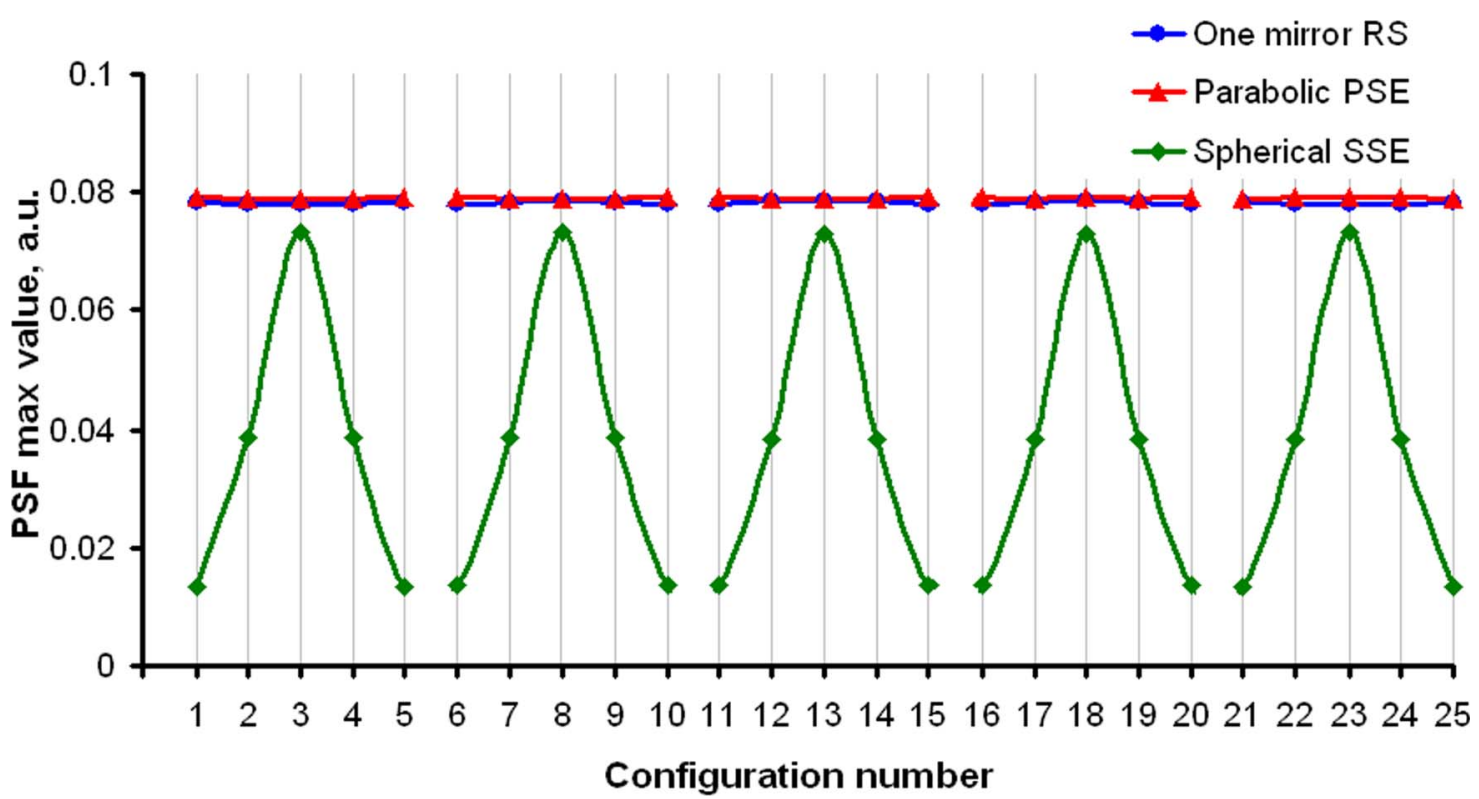

Fig. 12. (Color online) The variation in point spread function maxima for the 25 system configurations examined in each of the three scan engines.

and/or before the second scan mirror, as shown by Lindblom [26] at the expense of chromatic aberration.

The patent by Seel [3] would cover the PSE design described here as it has been broadly written in the claims, although the optical arrangement presented in that document and in Fig. 13 has the parabolic surface rotated, effectively inverting the sections used as off-axis parabolic reflectors. Additionally, the location and deflection axis of the scan mirrors guarantees the system will suffer larger aberrations than a spherical-mirror-based alternative and, therefore, this design is inappropriate as an optical relay.

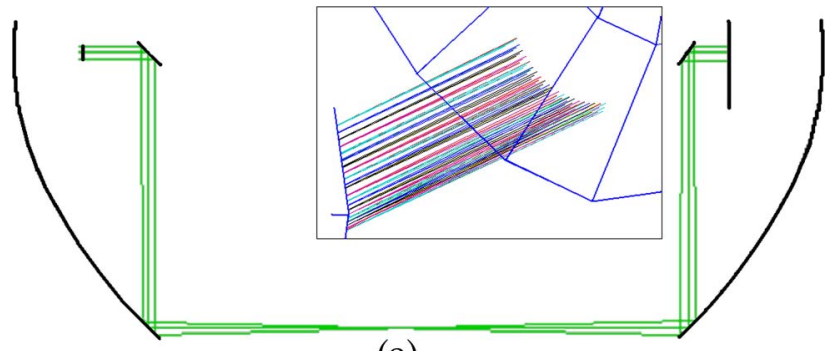

(a)

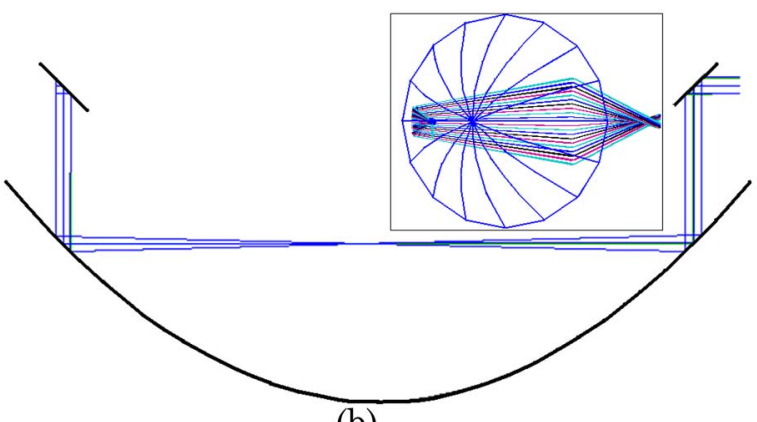

(b)

Fig. 13. (Color online) Comparison between (a) PSE and (b) Seel design. Insets show the curvature of scan lines reflecting from parabolic surfaces and the aberrations evident in the Seel approach.
The aberrations arise because only the beam that will come onto the optical surface parallel to the parabola's axis passes through the parabola's focus. When the first galvanometer is scanned, this condition is not met and a rather poor result emerges.

\section{Conclusion}

We have presented a design of an achromatic, afocal optical relay incorporating two off-axis parabolic reflectors and an appropriate rotational axis of the scan mirrors that eliminates line curvature in the scan pattern created. It exhibits good Gaussian spot size performance and excellent spot diagram and point spread function parameters for the range of scan angles evaluated. This system has been compared to a long-established standard optical relay design using spherical mirrors and found to be demonstrably superior in every metric. Additionally, the performance difference between the new design and an ideal single scan mirror is very slight. The spot diagrams and point spread functions show in stark detail an impressive performance advantage associated with the parabolic mirror design. This result should find immediate application in scanning microscopy and in the wider field of two-dimensional laser scanning.

Galiya Sharafutdinova is grateful to the University of Newcastle, Australia for her postgraduate research scholarship support.

\section{References}

1. A. Siegman, Lasers, 1st ed. (University Science, 1986).

2. J. Engelhardt, "Optical arrangement for deflecting a light beam, particularly in two substantially mutually perpendicular directions and confocal scanning microscope," U.S. patent 6,618,178 (9 September 2003).

3. M. Seel, "Beam deflection device," U.S. patent 6,433,908 (13 August 2002). 
4. M. Hara, "Optical-scanning microscope apparatus," U.S. patent 7,154,084 (2006).

5. K. Klose, "Application of additional mirrors for rectilinear laser scanning of wide formats," Appl. Opt. 17, 203-210 (1978).

6. M. Minsky, "Microscopy apparatus," U.S. patent 3,013,467 (19 December 1961).

7. W. B. Amos, "Achromatic scanning system," U.S. patent 4,997,242 (5 March 1991).

8. J. Montagu, "Galvanometric and resonant scanners," in Handbook of Optical and Laser Scanning G. F. Marshall, ed. (Marcell Dekker, 2004), pp. 417-476.

9. J. I. Montagu, "Scanning microscope employing improved scanning mechanism," U.S. patent 5,225,923 (6 July 1993).

10. L. Beiser and R. B. Johnson, "Devices, measurements and properties," in Handbook of Optics, 2 ed., M. Bass, ed. (McGraw-Hill, 1995).

11. B. Furlong and S. Motakef, "Scanning lenses and systems," Photonik Int. 3, 20-23 (2008).

12. W. Denk, J. P. Strickler, and W. W. Webb, "Two-photon laser microscopy," U.S. patent 5,034,613 (23 July 1991).

13. W. B. Wetherell, "Afocal systems," in Handbook of Optics, 2nd ed., M. Bass, ed. (Mc Graw-Hill, 1995), Chap. 2.

14. D. Malacara and Z. Malacara, Handbook of Optical Design, 2nd ed. (Marcel Dekker, 2004).

15. W. J. Smith, Modern Lens Design 2nd ed. (McGraw-Hill, 2005), p. 631.

16. M. v. Exter, C. Fattinger, and D. Grischkowsky, "Highbrightness terahertz beams characterized with an ultrafast detector," Appl. Phys. Lett. 55, 337-339 (1989).
17. D. Grischkowsky and M. van Exter, "Characterization of an optoelectronic terahertz beam system," IEEE Trans. Microwave Theory Tech. 38, 1684-1691 (1990).

18. D. Grischkowsky, S. Keiding, M. van Exter, and C. Fattinger, "Far-infrared time-domain spectroscopy with terahertz beams of dielectrics and semiconductors," J. Opt. Soc. Am. B 7, 20062015 (1990).

19. M. V. Mantravadi, D. H. Rose, J. T. Hall, and D. C. Richman, "Tilted primary clamshell lens laser scanner," U.S. patent 5,903,386 (11 May 1999).

20. W. B. Wetherell and M. P. Rimmer, "Confocal paraboloids: some comments," Appl. Opt. 13, 2192-2193 (1974).

21. H. Gross, F. Blechinger, and B. Achtner, Handbook of Optical Systems: Survey of Optical Instruments (Wiley-VCH, 2008), Vol. 4.

22. J.-C. Diels and W. Rudolph, Ultrashort Laser Pulse Phenomena: Fundamentals, Techniques, and Applications on a Femtosecond Time Scale, Optics and Photonics Series (Academic, 1996), p. 581.

23. J. G. White, "Method of operating a scanning confocal imaging system," U.S. patent 5,144,477 (1 September 1992).

24. W. B. Amos and J. G. White, "How the confocal laser scanning microscope entered biological research," Biol. Cell 95, 335342 (2003).

25. J. M. Howard and B. D. Stone, "Imaging a point with two spherical mirrors," J. Opt. Soc. Am. A 15, 3045-3056 (1998).

26. P. Lindblom, "Imaging system comprising a concave mirror," U.S. patent 6,965,483 (15 November 2005). 